\title{
Variações na produção de sementes e recomendações para o manejo de uso múltiplo da andirobeira
}

\author{
Helio Tonini ${ }^{1}$ \\ ${ }^{1}$ Embrapapa Pecuária Sul, Rodovia BR-153, Km 632,9, Vila Industrial, Zona Rural, CP. 242, CEP 96401-970, Bagé, RS, Brasil
}

*Autor correspondente:
helio.tonini@embrapa.br

Termos para indexação:

Carapa guianensis

Sementes oleaginosas

Manejo florestal

Index terms:

Carapa guianensis

Oilseeds

Forest management

Histórico do artigo:

Recebido em 04/07/2017

Aprovado em 01/12/2017

Publicado em 29/12/2017

doi: 10.4336/2017.pfb.37.92.1472

\begin{abstract}
Resumo - O objetivo deste trabalho foi avaliar a variação anual da produção de frutos da andirobeira em floresta nativa e as suas implicações para o manejo florestal de uso múltiplo. Os dados foram coletados em floresta nativa, no sul do Estado de Roraima em parcela permanente de 9 ha $(300 \mathrm{~m}$ x $300 \mathrm{~m})$ onde a produção de sementes de 115 árvores foi monitorada durante quatro anos. Observou-se que a produção de sementes da população variou entre os anos de observação, sendo maio a julho o período de maior produção. Poucos indivíduos $(22,6 \%)$ concentraram a maior parte da produção de sementes $(80,7 \%)$ e as árvores mais produtivas apresentaram diâmetro a $1,30 \mathrm{~m}$ do solo entre 40 a $70 \mathrm{~cm}$. Recomenda-se a adoção de um diâmetro mínimo de corte de 70 $\mathrm{cm}$ em planos de manejo madeireiros na região em estudo. Para o controle na coleta das sementes recomenda-se restringir a coleta aos indivíduos mais produtivos e somente no período de máxima frutificação.
\end{abstract}

\section{Annual variations in seed yield and implications for multiple use of crabwood}

\begin{abstract}
The objective of this study was to evaluate the annual variation in seed yield of crabwood, and discuss implications for multiple use management of the species. Data were collected in a forest area in the southern of Roraima State in a permanent plot of 9 ha $(300 \mathrm{~m} \times 300 \mathrm{~m})$ with a natural population of crabwood. Seed production was monitored of 115 trees during four years. Seed yield varied among years. May to July was the period of seed yeld. Few trees $(22.6 \%)$ concentrated the bigger part of seeds yield $(80.7 \%)$ and the most productive trees had diameter at $1.30 \mathrm{~m}$ above ground level between $40 \mathrm{~cm}-70 \mathrm{~cm}$. Therefore we recommend $70 \mathrm{~cm}$ as minimum cutting diameter for wood exploitation. For sustainable crabwood seed management we recommend to restrict seed collection from the most productive trees and only during the period of maximum seed dispersal.
\end{abstract}

\section{Introdução}

A andirobeira (Carapa guianensis Aubl.) é considerada uma espécie florestal amazônica com grande potencial para utilização em uso múltiplo em florestas naturais, sendo possível a sua utilização na produção de madeira e sementes de forma compatível, dependendo da estrutura da floresta, das taxas de exploração e do tipo florestal (Klimas et al., 2012).
É uma árvore da família meliácea que pode atingir até $55 \mathrm{~m}$ de altura. Possui madeira moderadamente pesada que permite bom acabamento, sendo muito procurada no mercado interno e externo para a fabricação de móveis, lâminas, compensados, caixotaria fina e acabamentos internos de barcos e navios (Loureiro et al., 1979).

O óleo extraído das sementes é utilizado pelas populações tradicionais na preparação de sabão e 
cosméticos, como repelente de insetos e tratamento de artrite, distensões musculares e alterações dos tecidos cutâneos (Plowden, 2004; Shanley, 2005). O óleo é comercializado em outras regiões do Brasil e exportado para uso na indústria de cosméticos da França, Alemanha e dos Estados Unidos (Gonçalves, 2001).

Carapa guianensis é considerada propícia para o manejo em florestas naturais, por ocorrer em abundância em diferentes tipos florestais e regiões da Amazônia; apresentar alta densidade com distribuição espacial agrupada e uma estrutura populacional favorável, típica de espécies com regeneração de sub-bosque tolerante à sombra (Henriques \& Souza, 1989; Hall, 1994; Peters, 1996; Klimas et al., 2007). Uma das características da espécie nas florestas amazônicas é apresentar estrutura do tipo $\mathrm{J}$ invertido, indicando grande estoque de indivíduos nas menores classes diamétricas e um recrutamento constante (Henriques \& Souza, 1989; Ramirez \& Arroyo, 1990; Peters, 1996; Klimas et al., 2007; Tonini et al., 2009).

No entanto, a irregularidade da produção de sementes é citada como entrave na comercialização da andiroba, uma vez que as árvores apresentam frutificação variável entre os anos e pouco se conhece sobre os padrões de frutificação da espécie (Forget, 1996; Dias et al., 2002; Balzon et al., 2004; Shanley, 2005).

O sistema de corte seletivo, comumente aplicado no manejo de florestas tropicais, é baseado em um diâmetro mínimo de corte (DMC), onde a densidade de indivíduos de espécies comerciais maiores do que um determinado diâmetro define a intensidade de corte. Este limite é estabelecido em função de demandas de mercado e, normalmente, não leva em consideração a biologia e a persistência das espécies exploradas. Segundo Sist et al. (2003), o DMC deve ser definido de acordo com a estrutura, a densidade e o diâmetro na idade reprodutiva.

Assim, o estudo sobre a frutificação da andirobeira e sua relação com a produção de sementes é fundamental para o manejo sustentável da espécie com finalidade madeireira e/ou não-madeireira, servindo como base para a definição do DMC e de diretrizes para a elaboração de políticas públicas relacionadas ao uso sustentável dos recursos florestais (Pena, 2007).

Neste contexto, o objetivo deste trabalho foi avaliar a variação anual da produção de frutos da andirobeira em floresta nativa e as suas implicações para o manejo florestal de uso múltiplo.

Pesq. flor. bras., Colombo, v. 37, n. 92, p. 563-568, out./dez. 2017

\section{Material e métodos}

\section{Área de estudo e amostragem}

O estudo foi realizado em Floresta Ombrófila Densa em área de terra firme (Brasil, 1975) com ocorrência natural de andiroba no sul do Estado de Roraima, no Município de São João da Baliza $\left(00^{\circ} 57^{\prime} 02^{\prime}\right.$ N e $59^{0} 54^{\prime} 41^{\prime \prime}$ 'W).

O clima na região é classificado por Köppen como Ami (tropical chuvoso com pequeno período de seca) com precipitação média anual entre $1.700 \mathrm{~mm}$ e $2.000 \mathrm{~mm}$. O período chuvoso ocorre com maior frequência de abril a agosto, com totais mensais superiores a $100 \mathrm{~mm}$. A partir de setembro há redução de precipitação, caracterizando o período seco que se estende até março. A temperatura média anual é $27{ }^{\circ} \mathrm{C}$ (Fundação do Meio Ambiente e Tecnologia de Roraima, 1994).

Os dados foram coletados em uma parcela permanente de 9 ha, localizada na área de reserva legal de uma propriedade particular de 100 ha. A parcela foi instalada pela Embrapa Roraima para a realização de estudos de ecologia e manejo de espécies de uso não madeireiro, como a castanheira-do-brasil (Bertholetia excelsa) e a andiroba (Carapa guianensis), no âmbito do Projeto Kamukaia. Nesta área, a densidade de andirobeiras é de 12,7 árvores ha-1 (Tonini et al., 2009).

Monitorou-se a produção de frutos e sementes de 115 árvores de 2006 a 2009. Em cada árvore, foi realizada a coleta e a pesagem das sementes em campo, com balança de gancho digital (precisão de $50 \mathrm{~g}$ ). A coleta foi quinzenal durante o período de queda dos frutos, que nesta região ocorre entre abril e agosto.

Para avaliar a produção por classe de tamanho, as árvores foram classificadas em quatro classes de produção (nula $=0 \mathrm{~kg} ; 0,1 \leq$ baixa $<50 \mathrm{~kg} ; 50 \leq$ média $<100 \mathrm{~kg}$ e alta $\geq 100 \mathrm{~kg}$ ) e três classes de tamanho em relação ao diâmetro a $1,30 \mathrm{~m}$ do solo (pequenas, DAP $<30 \mathrm{~cm}$; médias, $30 \mathrm{~cm} \leq \mathrm{DAP}<50 \mathrm{~cm}$; grandes, DAP $\geq 50 \mathrm{~cm})$.

Utilizou-se o teste de Kolmogorov-Smirnov para avaliar a normalidade dos dados de produção de sementes. Para a heterogeneidade de variância foi utilizado o teste de Levene.

Foi necessário utilizar testes não paramétricos para inferir sobre as relações entre as variáveis estudadas. Para verificar se existiam diferenças de produção entre 
árvores e/ou entre anos nas diferentes classes diamétricas, utilizou-se o teste de Kruskal-Wallis. Os contrastes entre grupos foram avaliados pelo teste de Mann-Witney, com a aplicação da correção de Bonferroni.

\section{Resultados}

Os dados de produção anual de sementes diferiram significativamente da distribuição normal para os anos de 2006 a 2009 ( $Z=0,322,0,298,0,304$ e 0,341, respectivamente, para $p=0,01)$, e apresentaram heterogeneidade de variância $\left(\mathrm{F}_{(3,456)}=14,65\right.$, para $\mathrm{p}=$ $0,01)$.

A média anual da produção de sementes por árvore adulta, com diâmetro a $1,30 \mathrm{~m}$ do solo $-(\mathrm{DAP}) \geq 30 \mathrm{~cm}$ foi $49,9 \mathrm{~kg}(\mathrm{~s}=68,6 ; \mathrm{n}=64)$ e a maioria dos indivíduos $(65,6 \%)$ produziram frutos todos os anos. As árvores com DAP $<30 \mathrm{~cm}$, consideradas juvenis (Tonini et al., 2009), produziram em média $3,96 \mathrm{~kg}$ de sementes ( $\mathrm{s}=$ $7,49, \mathrm{n}=49)$ e a maioria dos indivíduos $(61,2 \%)$ não produziu frutos durante o período de avaliação.

A produção de sementes da população variou entre os $\operatorname{anos}\left(\chi_{(3)}^{2}=8,1, p=0,044\right)$. A maior produção (Figura 1A) foi registrada em 2008, com $1.672,5 \mathrm{~kg}(185,8 \mathrm{~kg}$ $\left.\mathrm{ha}^{-1}\right)$. Neste ano, a produção por árvore variou de um mínimo de $200 \mathrm{~g}$ a um máximo de $180 \mathrm{~kg}$.

A análise da produção total e da proporção de indivíduos frutificando (Figura 1b) indicou que as árvores mais produtivas apresentaram diâmetro entre 60 $\mathrm{cm}$ a $70 \mathrm{~cm}$. Nesta classe, a produção média por árvore foi $151,3 \mathrm{~kg}$ com $100 \%$ dos indivíduos produzindo frutos todos os anos. A partir de $70 \mathrm{~cm}$ de DAP observou-se um declínio na produção, indicando senescência. A proporção de indivíduos frutificando foi maior para as árvores com DAP entre $40 \mathrm{~cm}$ a $90 \mathrm{~cm}$.

A distribuição de frequência para a população estudada (Figura 1c) foi do tipo hipérbole negativa ( $\mathrm{j}$ invertido) com predominância de árvores nas menores classes diamétricas.

A produção por classe de tamanho está apresentada na Figura 2. Somente as árvores grandes (DAP $\geq 50 \mathrm{~cm}$ ) apresentaram produção superior a $100 \mathrm{~kg}$ nos 4 anos de observação. Este grupo foi formado por apenas 8 árvores ( $6,9 \%$ do total) e foi responsável pela produção de $1.497,3 \mathrm{~kg}$ de sementes (44,6\% da produção total). Acrescentando-se as árvores médias, de produção média, o número de árvores aumentou para 26 , ou seja, $22,6 \%$ das árvores produziram $2.710,5 \mathrm{~kg}$ de sementes $(80,7 \%$ da produção total).
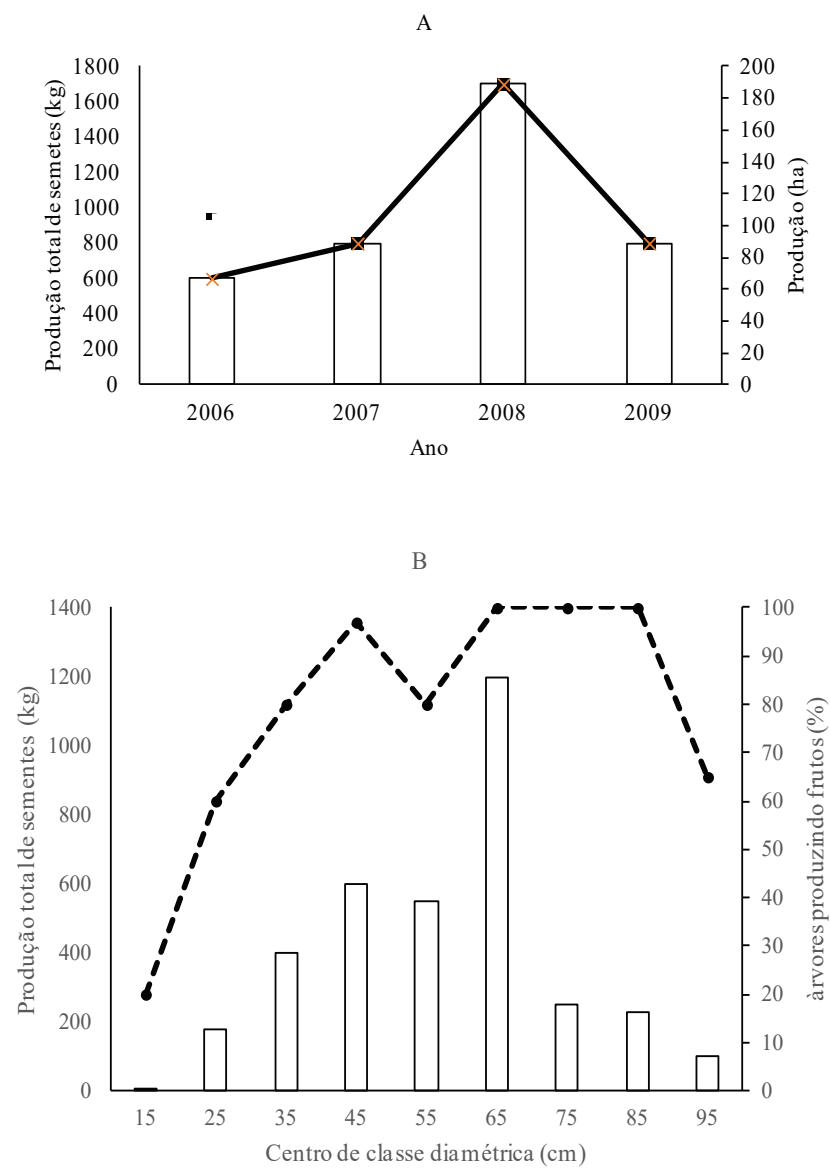

C

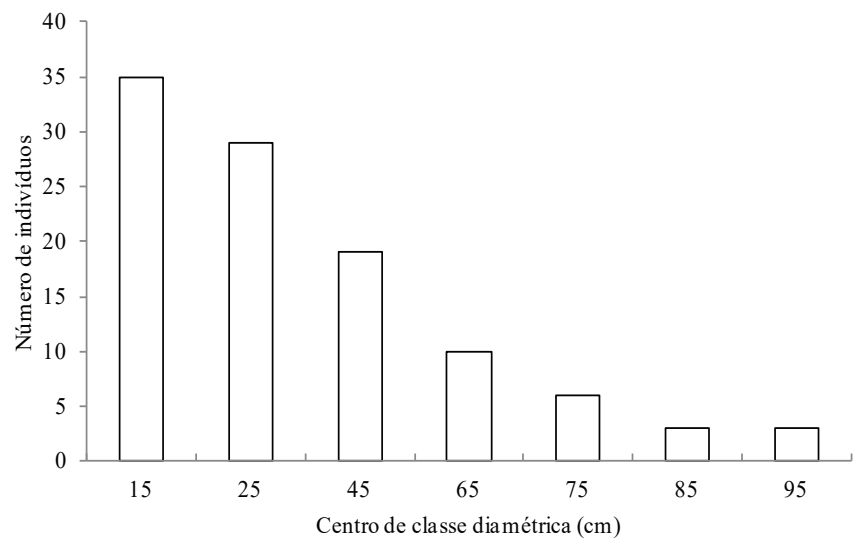

Figura 1. Produção total e media de peso fresco de sementes por hectare de 2006 a 2009 (A); Produção total de peso fresco de sementes e porcentagem de árvores em produção por classes de diâmetro (B); Número de indivíduos por classe diamétrica e diâmetro mínino de corte sugerido para a exploração madeireira (C). 


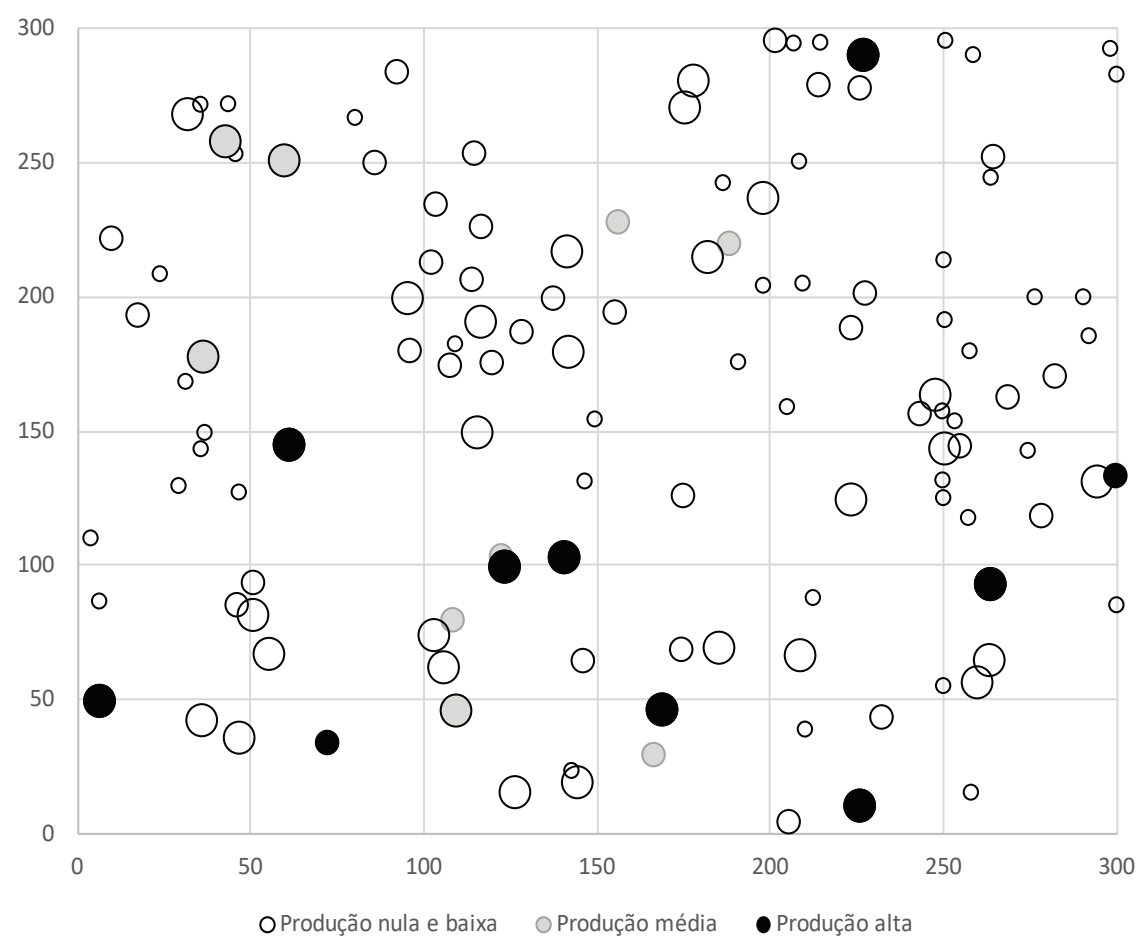

Figura 2. Distribuição espacial de andirobeiras em função do diâmetro a 1,30 m do solo (DAP) e da produção de sementes $(\mathrm{kg})$. Círculos pequenos indicam árvores pequenas (DAP $<30 \mathrm{~cm}$; círculos médios indicam arvores médias $30 \mathrm{~cm} \leq \mathrm{DAP}<$ $50 \mathrm{~cm}$ e os maiores árvores grandes (DAP $\geq 50 \mathrm{~cm})$.

A distribuição temporal da queda de frutos ao longo das safras de 2007, 2008 e 2009 (Figura 3), indicou que a duração média do período de dispersão dos frutos por árvore foi de 3,5 meses e ocorreu de abril a setembro. O ano de 2006, por apresentar uma pequena produção e o intervalo entre coletas ser maior, foi desconsiderado nesta análise.

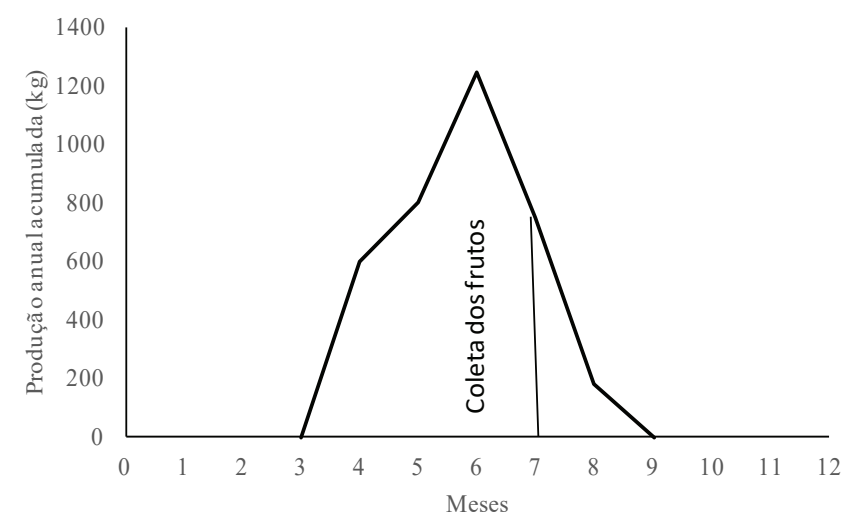

Figura 3. Distribuição da queda de sementes de Carapa guianensis (média de 2007 a 2009) em São João do Baliza, $\mathrm{RR}$, no período recomendado para a coleta dos frutos.

\section{Discussão}

Grandes variações anuais e diferenças regionais na produção de frutos e sementes parecem ser uma característica comum para o gênero Carapa na região amazônica e na América Central, conforme observado por MacHargue \& Hartshorn (1983) na Costa Rica e por Forget (1996) para Carapa procera na Guiana Francesa. A alta predação de sementes e plântulas, aliada à irregularidade na produção de frutos, parece ser importante no ciclo de vida da andirobeira e pode influenciar o sucesso de seu estabelecimento, recrutamento e regeneração natural (Pinto, 2007).

A produção observada em São João da Baliza $\left(49,9 \mathrm{~kg} \mathrm{arv}^{-1}\right)$, pode ser considerada alta, em comparação com a relatada por autores como Mellinger (2006), no Amazonas, e Plowden (2004), no sudeste do Pará, que observaram produção média de $7 \mathrm{~kg} \operatorname{arv}^{-1} \mathrm{e} 1,2 \mathrm{~kg} \operatorname{arv}^{-1}$, respectivamente. Produção mais alta foi observada por Rizzini \& Mors (1976), com arvores produzindo $180 \mathrm{~kg}$ a $200 \mathrm{~kg}$ de sementes ano ${ }^{-1}$, valores próximos à produção máxima observada por árvore em São João da Baliza.

Foram observados indivíduos frutificando em classes 
diamétricas a partir de $15 \mathrm{~cm}$ de diâmetro a $1,30 \mathrm{~m}$ do solo (DAP), estando acima do observado por Pena (2007) em estudo realizado em Beu Branco, PA, com $\mathrm{DAP}=11,7 \mathrm{~cm}$. A predominância de árvores pequenas na estrutura diamétrica da população, indicou que a espécie mantém um estoque suficientemente amplo de árvores finas para substituir as árvores de maior porte que venham a ser exploradas com fins madeireiros.

Considerando que as árvores mais produtivas apresentaram diâmetro entre $60 \mathrm{~cm}$ a $70 \mathrm{~cm}$ (Figura 1b), e que o diâmetro mínimo de corte (DMC) estabelecido pela Instrução Normativa do Ministério do Meio Ambiente $n^{\circ} 05$ de 2006 (Brasil, 2006) independentemente da espécie é de $50 \mathrm{~cm}$, pode-se prever que ao se cortar árvores com DAP a partir de 50 $\mathrm{cm}$, estarão sendo eliminadas as árvores que produzem as maiores quantidades de sementes, o que poderia impactar negativamente a regeneração natural e inviabilizar ecológica e economicamente o manejo florestal de uso múltiplo dessa espécie. Nas condições estudadas, o ideal seria permitir que fossem exploradas para fins madeireiros somente indivíduos com DAP $\geq 70 \mathrm{~cm}, o$ que ainda possibilitaria a exploração madeireira de uma árvore ha-1 (Figura 1c).

Ao estudar a viabilidade de combinar a exploração madeireira e não madeireira em andirobeiras nativas no Acre, Klimas et al. (2012) observaram que a exploração madeireira de $50 \%$ ou $100 \%$ das árvores com DAP $\geq 50$ (duas árvores ha ${ }^{-1}$ ) só seria compatível com uma taxa de coleta de apenas $10 \%$ das sementes em um ciclo de exploração madeireira de 50 anos.

Observou-se que a produção de sementes se concentrou em um número reduzido de indivíduos, o que facilita o esforço de coleta e pode reduzir os custos de exploração das sementes. Por outro lado, a coleta intensiva em poucos indivíduos, geralmente os mais produtivos, pode levar à seleção genética negativa, uma vez que os extrativistas tendem a concentrar a coleta nas árvores de maior produção (informação pessoal) e com isto deixar propágulos de árvores de menor produção.

Considerando as três formas de manejo de coleta de sementes propostas em Mellinger (2006) que consistem em: 1) alternância de áreas entre anos, 2) alternância de meses no mesmo ano e 3) ajustes na porcentagem de coleta, considerou-se que a alternância de áreas entre os anos não seria a mais indicada pela irregularidade e variabilidade da produção e pelo baixo número de indivíduos de grande produção, ou seja, a divisão da área poderia levar à grande irregularidade no fluxo de produção. $\mathrm{O}$ ajuste no percentual de coleta é de difícil aplicação prática, além de exigir grande conscientização por parte dos extrativistas.

Portanto, a opção de coletar as sementes nos indivíduos mais produtivos $(22,6 \%$ dos indivíduos) apenas nos meses que concentram a maior produção de sementes, de maio a julho (Figura 3 ) pode ser recomendada como técnica de manejo, pois permitiria saciar a fauna local, sem influenciar negativamente a qualidade genética e os índices de regeneração natural da população.

Simulando este cenário, obteve-se uma redução na produção de sementes (por renuncia), de $949,4 \mathrm{~kg} \mathrm{ha}^{-1}$ ou $105,5 \mathrm{~kg} \mathrm{ha}^{-1}$ de sementes, que representou aproximadamente $30 \%$ da produção total, ou seja, seriam coletadas, no máximo, $70 \%$ das sementes na área, sendo o restante deixado para a fauna e a regeneração natural.

Em comparação com o resultado obtido por Klimas et al. (2007) em floresta nativa no Acre, esta taxa de coleta seria muito elevada, indicando que deve ser ajustada para cada região, com base em levantamento prévio e monitoramento da população por longos períodos. Também indica que o procedimento aqui proposto deve ser testado em relação ao seu impacto na regeneração natural, sendo recomendável restringir ainda mais o período e a intensidade de coleta se o impacto for negativo.

A floresta estudada por Klimas et al. (2012) conforme Klimas et al. (2007), é mais densa em andirobeiras (25,7 arvores ha ${ }^{-1}$ com DAP $\geq 10 \mathrm{~cm}$ ) do que a floresta em São João da Baliza (12,7 arvores ha ${ }^{-1}$ com DAP $\geq 10$ $\mathrm{cm})$, porém, o número de árvores grandes (DAP $\geq 50$ $\mathrm{cm})$ é menor (3,2 arvores ha-1 contra 4,2 arvores ha-1), com poucos indivíduos com DAP $\geq 70 \mathrm{~cm}(0,1$ árvores ha $^{-1}$ contra 1,3 arvores ha $\left.{ }^{-1}\right)$. Portanto, o diâmetro médio de corte aqui proposto não poderia ser aplicado nesta floresta no Acre. No estudo de Klimas et al. (2012) foi considerada a exploração madeireira de duas arvores $\mathrm{ha}^{-1}$ (DAP $\geq 50 \mathrm{~cm}$ ), provavelmente, árvores com alta produção de sementes, o que pode ter tido grande influência no resultado obtido pelos autores.

\section{Conclusões}

A produção de sementes foi concentrada em poucos indivíduos e as árvores mais produtivas apresentaram diâmetro a $1,30 \mathrm{~m}$ da altura do solo entre $40 \mathrm{~cm}$ a $70 \mathrm{~cm}$. 
Com base na produção de sementes por classe diamétrica, recomenda-se adoção de um diâmetro mínimo de corte de $70 \mathrm{~cm}$, e a coleta das sementes apenas nos indivíduos mais produtivos no período de máxima frutificação.

\section{Agradecimentos}

Os autores agradecem ao CNPq, processo 575393/2008-4 pelo auxílio financeiro e a equipe do projeto Kamukaia-Manejo de Produtos Florestais Não Madeireiros na Amazônia, especialmente aos colegas Adebaldo Sampaio Teles e José de Anchieta Moreira da Costa pela dedicação e esmero na coleta dos dados.

\section{Referências}

Balzon, D. R. et al. Aspectos mercadológicos de produtos florestais não-madeireiros- análise retrospectiva. Floresta, v. 34, n. 3, p. 363 371, 2004. DOI: 10.5380/rf.v34i3.2422.

Brasil. Departamento Nacional da Produção Mineral. Projeto RADAMBRASIL. Folha NA.20 Boa Vista e parte das folhas NA21, Tumucumaque,NB.20 Roraima e NB 21: geologia, geomorfologia, pedologia, vegetação e uso potencial da terra. Rio de Janeiro, 1975. $428 \mathrm{p}$.

Brasil. Ministério do Meio Ambiente. Instrução Normativa $n^{\circ} 05$ de 11 de dezembro de 2006. Dispõe sobre os procedimentos técnicos para elaboração, apresentação, execução e avaliação dos Planos de Manejo Florestal Sustentável-PMFS nas florestas primitivas e suas formas de sucessão na Amazônia Legal e dá outras providências. Diário Oficial [da] República Federativa do Brasil, Brasília, DF, 13 dez. 2006.

Dias, A. S. et al. Manejo florestal diversificado em uma comunidad ribereña de la Amazônia brasileña. Revista Forestal Centroamericana, v. 38, p. 78-84, 2002.

Forget, P. M. Removal of seeds Carapa procera (Meliaceae) by rodents and their fate in rainforest in French Guiana. Journal of Tropical Ecology, v. 12, p. 751-761, 1996.

Fundação do Meio Ambiente e Tecnologia de Roraima. Roraima, o Brasil do hemisfério norte. Boa Vista: Ambitec, 1994. 512 p.

Gonçalves, V. A. Levantamento de mercado de produtos florestais não-madeireiros. Santarém: IBAMA, 2001. 65 p.

Hall, P. et al. Genetic diversity and mating system in a tropical tree, Carapa guianensis (Meliaceae). American Journal of Botany, v. 81, n. 9, p. 1104-1111, 1994.

Henriques, R. P. B. \& Sousa, E. C. E. G. Population structure, dispersion and microhabitat regeneration of Carapa guianensis in Northeastern Brazil. Biotropica, v. 21, n. 3, p. 204-209, 1989.
Klimas, C. A. et al. Population structure of Carapa guianensis in two Forest types in the southweastern Brazilian Amazon. Forest Ecoly and Managment, v. 250, p. 256-265, 2007.

Klimas, C. A. et al. Viability of combined timber and nontimber harvests for one species: a Carapa guianensis case study. Ecological Modelling, v. 246, p. 147-156, 2012. DOI: 10.1016/j. ecolmodel.2012.07.022

Loureiro, A. A. et al. Essências madeireiras da Amazônia. Manaus: INPA, 1979. v. 2. 187 p.

MacHargue, L. A. \& Hartshorn, G. S. Seed and seedling ecology of Carapa guianensis. Turrialba, v. 33, n. 4, p. 399-404, 1983.

Mellinger, L. L. Aspectos da regeneração natural e produção de sementes de Carapa guianensis Aubl. (andiroba) na Reserva de Desenvolvimento Sustentável Amanã (AM). 2006. 81 f. Dissertação (Mestrado em Ciências Biológicas) - Instituto Nacional de Pesquisas Amazônicas/Universidade Federal do Amazonas, Manaus.

Pena, J. W. P. Frutificação, produção e predação de sementes de Carapa guianensis AUBL. (Meliaceae) na Amazônia Oriental Brasileira. 2007. 60 f. Dissertação (Mestrado em Ciências Florestais) - Universidade Federal Rural da Amazônia, Belém.

Peters, C. M. Aprovechamiento sostenible de recursos no maderables en bosque húmedo tropical: un manual ecológico. Washington, DC: El Programa de Apoyo a la Biodiversidad, 1996. $51 \mathrm{p}$.

Pinto, A. A. Avaliação de danos causados por insetos em sementes de Andiroba (Carapa guianensis Aubl.) e Andirobinha ( $C$. procera DC.) (Meliaceae) na Reserva Florestal Adolpho Ducke em Manaus, AM, Brasil. 2007. 60 f. Dissertação (Mestrado em Biologia) - Instituto Nacional de Pesquisas da Amazônia, Manaus.

Plowden, C. The Ecology and harvest of andiroba seeds for oil production in the Brazilian Amazon. Conservation \& Society, v. 2, n. 2, p. 251-270, 2004.

Ramirez, N. \& Arroyo, M. K. Estructura poblacional de Copaifera pubiflora Benth. (Leguminosae; Caesalpinoideae) en los Altos Llanos Centrales de Venezuela. Biotropica, v. 22, n. 2, p. 124-132, 1990.

Rizzini, C. T \& Mors, W. B. Botânica econômica brasileira. São Paulo: EPUSP, 1976. 207 p.

Shanley, P. Andiroba (Carapa guianensis, Aubl.). In: Shanley, P. \& Medina, G. Frutíferas e plantas úteis na vida amazônica. Belém: Cifor, 2005. p. 41-50.

Sist, P. et al. Towards sustainable management of mixed dipterocarp forests of Southeast Asia: moving beyond minimum diameter cutting limits. Environmental Conservation, v. 30, n. 4, p. 364-374, 2003.

Tonini, H. et al. Estrutura, distribuição espacial e produção de sementes de andiroba (Carapa guianensis Aubl.) no sul do estado de Roraima. Ciência Florestal, v. 19, n. 2, p. 1-6, 2009. DOI: 10.5902/19805098413. 